\title{
Article \\ Adherence to the Japanese Food Guide: The Association between Three Scoring Systems and Cardiometabolic Risks in Japanese Adolescents
}

\author{
Masayuki Okuda ${ }^{1, *(\mathbb{D})}$, Aya Fujiwara ${ }^{2,3}$ (1) and Satoshi Sasaki ${ }^{3}$ \\ 1 Graduate School of Sciences and Technology for Innovation, Yamaguchi University, 1-1-1 Minami-Kogushi, \\ Ube 755-8505, Japan \\ 2 Department of Nutritional Epidemiology and Shokuiku, The National Institutes of Biomedical Innovation, \\ Health and Nutrition, 1-23-1 Toyama, Shinjuku-ku, Tokyo 162-8636, Japan; fujiwaraay@nibiohn.go.jp \\ 3 Department of Social and Preventive Epidemiology, Graduate School of Medicine and School of Public \\ Health, The University of Tokyo, 7-3-1 Hongo, Bunkyo-ku, Tokyo 113-0033, Japan; stssasak@m.u-tokyo.ac.jp \\ * Correspondence: okuda@yamaguchi-u.ac.jp; Tel.: +81-836-22-2231
}

check for

updates

Citation: Okuda, M.; Fujiwara, A.;

Sasaki, S. Adherence to the Japanese Food Guide: The Association between Three Scoring Systems and Cardiometabolic Risks in Japanese Adolescents. Nutrients 2022, 14, 43. https://doi.org/10.3390/nu 14010043

Academic Editors: Clare Collins and George Moschonis

Received: 2 December 2021

Accepted: 20 December 2021

Published: 23 December 2021

Publisher's Note: MDPI stays neutral with regard to jurisdictional claims in published maps and institutional affiliations.

Copyright: (c) 2021 by the authors. Licensee MDPI, Basel, Switzerland. This article is an open access article distributed under the terms and conditions of the Creative Commons Attribution (CC BY) license (https:/ / creativecommons.org/licenses/by/ $4.0 /)$.

\begin{abstract}
The Japanese Food Guide Spinning Top (JFGST) indicates optimal intake of five food groups (grain, fish and meat, vegetables, milk, and fruits) and sugar and confectionaries. We aimed to investigate whether adherence to the JFGST in 8th grade junior high school students $(n=3162)$ was associated with cardiometabolic risks and how different scorings of the JFGST influenced the associations. Metabolic risks were assessed from anthropometrics, blood pressure measurements, and blood glucose and lipid profile measurements. Three types of scoring adherent to the JFGST were analyzed (10 points were given for each item with optimal intake; range: 0-60): the original scoring (ORG scoring); first modified scoring, which had no upper limits for vegetables and fruits (MOD1 scoring); and MOD2 scoring without upper limits for five dishes (MOD2 scoring). The MOD2 scoring was positively associated with dietary fiber, potassium, calcium, and vitamins. All types of scorings were associated with low glucose levels ( $p \leq 0.001)$; the MOD2 scoring was associated with low systolic blood pressure $(p=0.001)$ and low cardiometabolic risk $(p=0.003)$. Our findings suggest that Japanese adolescents adherent to the JFGST had low cardiometabolic risks and should not fall below lower limits for intake of the abovementioned five food groups.
\end{abstract}

Keywords: adolescents; blood pressure; cardiometabolic risks; diet quality; energy-providing nutrients; fasting plasma glucose level; Japan Food Guide Spinning Top; Shokuiku

\section{Introduction}

Numerous dietary indices, including modifications and variants, have been proposed to assess overall diet quality representing nutrient recommendations, healthy dietary habits, and dietary variety [1]. People consume a combination of several foods containing various nutrients that are interrelated metabolically and functionally in one sitting. Dietary indices have been reported to be associated with health outcomes, such as total mortality and cardiovascular diseases [2]. Most dietary indices are based on dietary guidelines and food guides in the US, such as the Healthy Eating Index and the Diet Quality Index [3,4], or on a Mediterranean diet pattern, such as the Mediterranean Diet Score and the Mediterranean Diet Quality Index for children and adolescents $[5,6]$. Dietary patterns in these countries are different from those of Japan. Fat and carbohydrate in food supply accounted for 37.7-43.8\% and $43.3-48.7 \%$ of the total energy, respectively, in 2017 in North America, Western Europe, and Australia. In contrast, the proportions of these nutritional components were 29.8-30.3\% and 56.7-57.3\%, respectively, in 2017-2019 in Japan [7]. Japanese people may consume less fat and more carbohydrates than people in countries where the aforementioned dietary indices are used. The longevity and relatively low morbidity of cardiovascular diseases in Japan are attributed to the Japanese diet. Appropriate dietary indices associated with 
Japanese health should be used to assess Japanese dietary patterns. Since high carbohydrate intake is considered a feature of the Japanese diet [8], a diet index comprising energyproviding nutrients could be applied to achieve health-promoting benefits.

The Japanese Food Guide Spinning Top (JFGST) was developed in 2005 as an educational tool to promote healthy behavior in Japanese people [9]. It was based on the Dietary Guidelines for Japanese and the Dietary Reference Intakes for Japanese, which take into account the Japanese culture, that is, considering carbohydrate intake. The JFGST is applicable for individuals aged 6 years or older on the assumption that they adhere to their estimated energy requirements. The JFGST is expected to be available in food and nutrition education ("Shokuiku") campaign.

JFGST-based scoring has been used to assess diet quality in several reports. Adherence to the JFGST showed beneficial associations with the scores on mortality, metabolic risk factors, depression, and sleep [10-20]. However, scoring methods based on the JFGST vary among reports, that is, with or without upper limits of the recommended range of food groups [10-12]; with additional scoring items, such as energy [10,11], sodium intake [12], and red/white meat [11]; and using continuous points [12] or discrete points after rounding to whole numbers [16]. Different scoring methods may influence associations with health outcomes in different ways.

The original JFGST comprises five food groups with upper and lower limits using discrete points, and two items with upper limits, including sugar and confectionaries, and alcohol beverages. However, it does not include items on energy, sodium, or red and white meat [9]. Since there were few habitual consumers of alcohol beverages in Japanese adolescents (0.5-1.8\%) [21], six items, including five food groups and sugar and confectionaries, are appropriate. Meanwhile, achieving dietary and nutritional balance is an essential goal of the JFGST [9]. In the Dietary Reference Intakes for Japanese, items with defined upper limits for preventing lifestyle-related diseases are sodium and saturated fatty acids [22]. However, these could not determine the upper limits of vegetable dishes, fish and meat dishes, milk, and fruits. Oba et al. used the original lower and upper limits of the JFGST [10], Kurotani et al. removed the upper limits for vegetable dishes and fruits [11], and Kuriyama et al. did not use the upper limits for five food groups [12]. While only the score without the upper limits was associated with favorable nutrient intake patterns in Japanese women [12], all three scoring systems showed inverse associations with mortality $[10,11]$ and metabolic risk factors $[15,17]$. In addition, there are no reports that the scores were coincidentally calculated with or without upper limits of the food groups to assess the associations with metabolic factors except for one study on adults, which showed that both scorings had unexpected associations with serum cholesterols and glycosylated hemoglobin [17].

Previous reports based on the JFGST targeted adult population [10-18,20], except for one study that analyzed a population aged < 6 years [19], which is not a target age population of the JFGST. However, there are no reports for adolescents. Therefore, the association between metabolic risk factors and the scoring system in adolescents is still unknown. The aim of the study was to investigate the association between diet quality score of adherence to the JFGST, representing a feature of Japanese diet, and metabolic risk factors in Japanese adolescents. We examined three scoring systems to clarify the different factors that influence the associations between the scores and cardiometabolic risk factors.

\section{Materials and Methods}

\subsection{Participants}

This study was part of the Shunan Child Cohort Study described in detail elsewhere [23-25]. The participants were 8th-grade junior high school students from 17 junior high schools in Shunan City, Japan. From 6805 students attending any of the schools between 2006 and 2010, 6226 students participated in this study with their guardians' consent. We excluded those with missing variables; with physician-diagnosed diseases; who had taken breakfast before blood extraction; with high plasma glucose, triglyceride, or 
low-density lipoprotein cholesterol levels due to possible postprandial values, or familial hypercholesterolemia; and with implausible energy intake (Figure 1). Overall, we analyzed the data of 3162 participants.

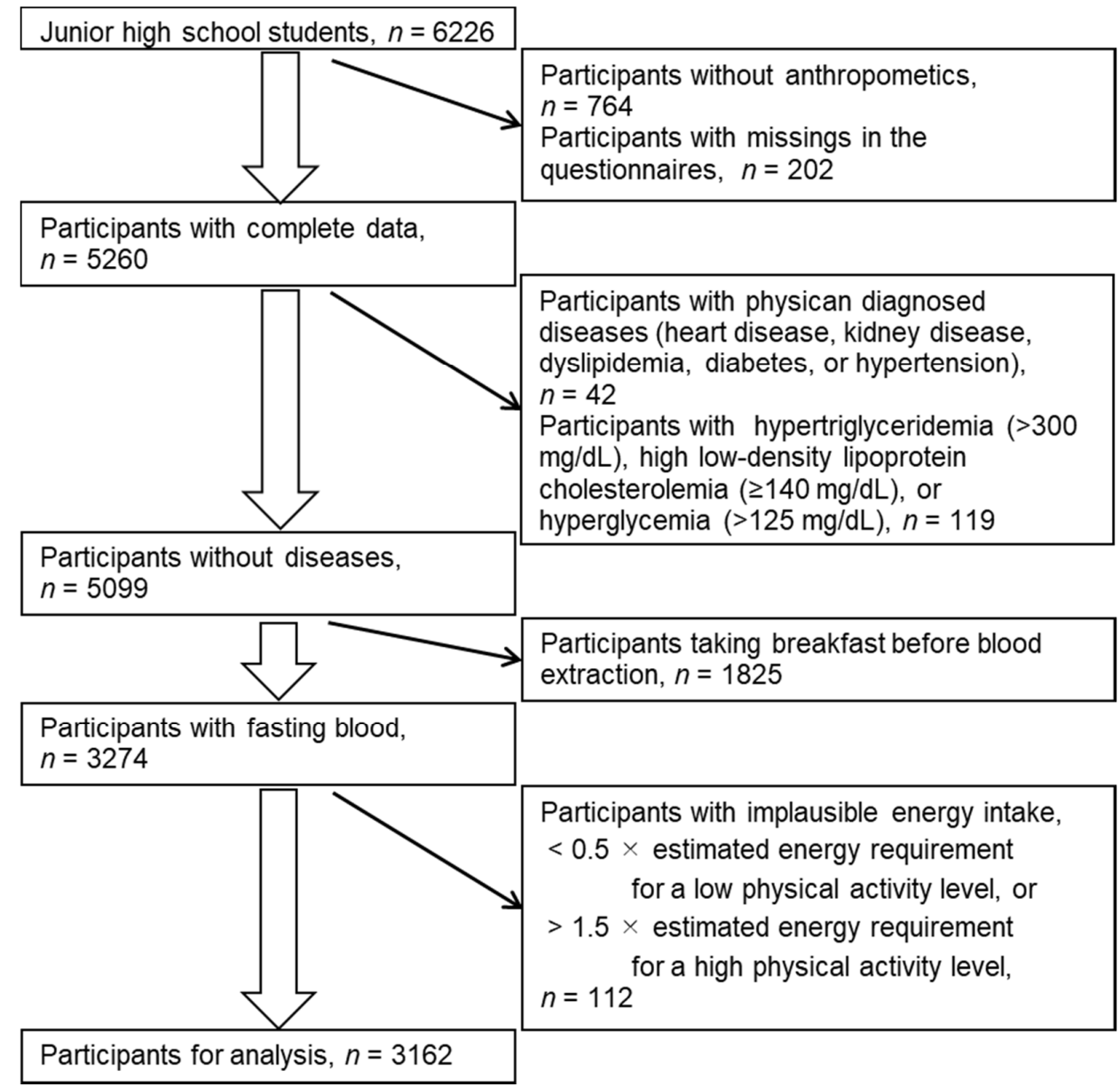

Figure 1. Selection of participants for analysis.

\subsection{Dietary Assessment}

Foods and nutrients were assessed using a brief-type self-administered diet history questionnaire for youths (BDHQ15y). The BDHQ15y, which assesses the consumption frequency of 63 selected food items and 17 dietary behaviors in the previous month, is a modification of the BDHQ for adults. The correlation coefficients between the estimates from the single BDHQ for adults and 16-day dietary records were 0.17-0.66 (Spearman correlation) for cereals, sugar and confectionaries, vegetables, potatoes, fruits, fish, meat, egg, dairy products, and non-alcoholic beverages [26]; 0.35-0.64 (Pearson correlation) for protein, fat, and carbohydrate; $0.44-0.66$ (Pearson correlation) for sodium, potassium, calcium, magnesium, and iron; and 0.42-0.63 (Pearson correlation) for beta-carotene equivalent, and vitamin C [27]. Spearman correlation coefficients with corresponding biomarkers in adolescents were $0.26-0.31$ for serum carotenoids; $0.22-0.48$ for red blood corpuscle marine omega-3 polyunsaturated fatty acids [28]; 0.11-0.30 for urinary nitrogen [29]; and 0.05 for sodium; 0.11 for potassium; and 0.10 for the sodium-to-potassium ratio [30]. Plausible responders were considered to have energy intake $\geq 0.5$ and $\leq 1.5$ times of age- and sexspecific estimated energy requirements for low and high physical activity levels (PALs), respectively [31]. Intake of nutrients and food was adjusted using an energy density method [32]. As we could not determine the PAL of the participants, individual intake was standardized assuming energy intake equal to age- and sex-specific estimated energy requirements for moderate PAL. 
Diet quality was assessed from five food dish groups (grain dishes, vegetable dishes, fish and meat dishes, milk, and fruits) and sugar and confectionaries based on the JFGST (Supplementary Table S1) $[9,33]$. The original score was calculated from the standardized energy-adjusted nutrient and food intake based on the serving standards of the JFGST scoring system. For five dishes, one serving (SV) corresponded to $40 \mathrm{~g}$ of carbohydrate for grains, $70 \mathrm{~g}$ of vegetables for vegetable dishes, $6 \mathrm{~g}$ of protein for fish and meat, $100 \mathrm{mg}$ of calcium for milk, and $100 \mathrm{~g}$ of fruits for fruits. The number of servings was rounded to whole numbers as follows: if the value obtained was between 0.67 and $<1.5$, it was counted as one serving; any value between 1.5 and $<2.5$ was rounded off to two servings; and any value between 2.5 and $<3.5$ was rounded off to three servings. The rounding-off manner applies to the succeeding values (i.e., 3.5 to $<4.5$ and so on). The maximum point per dish was 10 . When the intake was lower or higher than the optimum, a point was calculated as SV/lower limit of the optimum $\times 10$ or $10-(\mathrm{SV}-$ the upper limit)/upper limit $\times 10$, respectively (Supplementary Table S1). When the calculated point was less than zero, zero point was given. Optimum intake of sugar and confectionaries, which are not counted in SV, was $<200 \mathrm{kcal}$ giving 10 points, and points for $\geq 200 \mathrm{kcal}$ were calculated (corresponding energy -200 ) $/ 200 \times 10$. When the calculated point was less than zero, zero point was given. The first modified score (MOD1 score) was the same as the original score (ORG score), except that no upper limits were set for vegetable dishes and fruits according to Kurotani et al. $[10,11]$. The second modified score (MOD2 score) had no upper limits for the five dishes according to Kuriyama et al. [12]. The scores of three scoring methods ranged from 0 to 60 , with higher values indicating higher adherence to the JFGST.

\subsection{Cardiometabolic Risk Factors}

School nurses measured the participants' body height and weight near $0.1 \mathrm{~cm}$ and $0.1 \mathrm{~kg}$, respectively. Body mass index (BMI; $\mathrm{kg} / \mathrm{m}^{2}$ ) was calculated as the weight divided by the square of height. The standard deviation score of BMI (zBMI) was calculated based on the Japanese reference in 2000 [34] using the Lambda-Mu-Sigma method [35]. Blood pressure (BP) was measured twice using auto-sphygmomanometers (HEM-707, HEM-757, or HEM-780, OMRON, Kyoto, Japan) after 5 min of sitting. Mean systolic and diastolic blood pressure (SBP and DBP, respectively) measurements were used. The participants were asked to fast 10-12 h before the blood extraction. Triglyceride (TG), low-density and high-density lipoprotein serum cholesterol (LDL-C and HDL-C, respectively), and plasma glucose levels were measured from blood specimens. Cardiometabolic risks were defined based on the definition of the International Diabetes Federation (32): zBMI $\geq 1$; $\mathrm{TG} \geq 150 \mathrm{mg} / \mathrm{dL}$; LDL-C $\geq 120 \mathrm{mg} / \mathrm{dL}$; HDL-C $<40 \mathrm{mg} / \mathrm{dL}$; fasting plasma glucose $\geq 100 \mathrm{mg} / \mathrm{dL}$; and $\mathrm{SBP} \geq 130 \mathrm{mmHg}$ and/or DBP $\geq 85 \mathrm{mmHg}$. Cardiometabolic risks were summed up as a metabolic syndrome score (MS score) ranging from 0 to 6.

\subsection{Confounding Factors}

The lifestyle questionnaire assessed movement behavior and household information. Sports activity ( $>2$ times/week or not), TV watching ( $>2 \mathrm{~h} / \mathrm{d}$ or not), sleep duration $(\mathrm{h})$, number of siblings $(1,2$, or $\geq 3$ ), and single parent (yes or no) were used as possible confounders.

\subsection{Statistical Analysis}

Unless specified, variables are expressed as mean \pm standard deviation or count (\%). After checking for normality using quantile-quantile plots, the JFGST score points and triglyceride levels are expressed as median (minimum, maximum). Scores were categorized based on quintile levels (the lowest Q1 to the highest Q5). The median of energy-adjusted food and nutrient intake (\% of total energy intake for energy-providing nutrients and amount per $1000 \mathrm{kcal}$ for remaining nutrients and food groups) in each category and the ratio of intake in Q5:Q1 (\%) were calculated to examine the contribution of foods and nutrients to the score. Non-parametric trend tests for food and nutrients across Q1 to 
Q5 were performed using the Jonckheere-Terpstra test for two-sided monotonic trends. Gaussian linear regression models were used to examine the association between the score and individual cardiometabolic risks. When the triglyceride level was used as a dependent variable, it was natural log transformed. Poisson regression models were used for the MS score because it had a skewed distribution of zero (Table 1); the effect on the dependent variable was an exponential function of a coefficient. Coefficients of both regression models were calculated as an effect of 10 points of score. $\mathrm{R}$ version 4.0.3 (R Foundation for Statistical Computing) was used [36], and the significance level was set at $p=0.05$.

Table 1. Characteristics of the analyzed participants $(n=3162)$.

\begin{tabular}{cc}
\hline Characteristics & Value \\
\hline Age, years & $13.6 \pm 0.3$ \\
Body mass index (BMI), $\mathrm{kg} / \mathrm{m}^{2}$ & $19.2 \pm 2.6$ \\
Z-score of BMI & $-0.2 \pm 0.9$ \\
Triglyceride, $\mathrm{mg} / \mathrm{dL}$ & $52(15,249)$ \\
LDL-C, $\mathrm{mg} / \mathrm{dL}$ & $88.1 \pm 19$ \\
HDL-C, mg/dL & $67.7 \pm 13.9$ \\
Fasting plasma glucose, $\mathrm{mg} / \mathrm{dL}$ & $90 \pm 5.8$ \\
Systolic blood pressure, $\mathrm{mmHg}$ & $114.2 \pm 11.6$ \\
Diastolic blood pressure, $\mathrm{mmHg}$ & $68.1 \pm 8.7$ \\
Energy intake, kcal/day & $2220 \pm 632$ \\
Sex & \\
Male & $1627(51.5)$ \\
Female & $1535(48.5)$ \\
MS score & \\
0 & $2298(72.7)$ \\
1 & $684(21.6)$ \\
2 & $150(4.7)$ \\
3, or 4 & $30(1.0)$
\end{tabular}

Data are expressed as mean \pm standard deviation, median (minimum, maximum), or count (\%). LDL-C, lowdensity lipoprotein cholesterol; HDL-C, high-density lipoprotein cholesterol. The MS score (metabolic syndrome score) is the clustering of cardiometabolic risks.

\section{Results}

The mean age of the participants was $13.6 \pm 0.3$ years, and the average BMI was $19.2 \pm 2.6 \mathrm{~kg} / \mathrm{m}^{2}$ (Table 1 ). Of 3162 participants, $27.3 \%$ had one or more cardiometabolic risk factors.

For many students, the servings of fish and meat dishes and milk were over the upper limits (70.2\% and 52.3\%, respectively), and some of them had zero points for these dishes (11.7\% and $41.1 \%$ of total participants, respectively; Supplementary Figures S1-S3). Therefore, the median points for fish and meat dishes and milk using the scoring with the upper limits (6.7 and 5 points, respectively) increased when using the scoring without the upper limits (10 and 10 points, respectively; Table 2). For less than 10\% of the students, the points for grain dishes (3.4\%), vegetable dishes $(9.7 \%)$, and fruits $(4.3 \%)$ were above the upper limits. The points for these dishes were similar regardless of whether the upper limits were applied or not. The median JFGST scores were 32 (minimum: 6.7, maximum: 58.3) using the ORG scoring, $33(3.7,58.8)$ using the MOD1 scoring with no upper limits for vegetable dishes and fruits, and $40.9(15,60)$ using the MOD2 scoring with no upper limits for all dishes except for sugar and confectionaries. 
Table 2. Diet quality scoring based on the Japanese Food Guide Spinning Top for adolescents $(n=3162)$.

\begin{tabular}{|c|c|c|c|c|c|c|c|c|c|}
\hline & \multicolumn{3}{|c|}{$\begin{array}{l}\text { Applying Both Upper } \\
\text { and Lower Limits }\end{array}$} & \multicolumn{3}{|c|}{$\begin{array}{c}\text { Applying } \\
\text { Only the Lower Limits }\end{array}$} & \multicolumn{3}{|c|}{$\begin{array}{c}\text { Applying } \\
\text { Only the Upper Limits }\end{array}$} \\
\hline & Median & Min. & Max. & Median & Min. & Max. & Median & Min. & Max. \\
\hline Grains & 8.3 & 2 & 10 & 8.3 & 2 & 10 & - & - & - \\
\hline Vegetables & 6.7 & 0 & 10 & 6.7 & 0 & 10 & - & - & - \\
\hline Fish and meat & 6.7 & 0 & 10 & 10 & 0 & 10 & - & - & - \\
\hline Milk & 5 & 0 & 10 & 10 & 0 & 10 & - & - & - \\
\hline Fruits & 5 & 0 & 10 & 5 & 0 & 10 & - & - & - \\
\hline Sugar and confectionaries & - & - & - & - & - & - & 0 & 0 & 10 \\
\hline
\end{tabular}

Min., minimum; Max., maximum.

Using the MOD2 scoring, the higher the total score, the higher the median intake of fish and meat, fish, and milk (Q5:Q1 ratios, 122-282\%), whereas other scorings showed opposite trends of the medians across the quintile categories in these food groups (Q5:Q1 ratios, 62-83\%; Table 3). In addition, the MOD2 scoring had higher Q5:Q1 ratios for vegetables and fruits than other scorings. In terms of nutrients, protein, sodium, and calcium intake showed opposite trends of the medians across the quintile categories in the MOD2 scoring and other scorings (Table 4); the higher score of the MOD2 scoring, the higher median intake of protein, sodium, and calcium. Beneficial health nutrients, such as dietary fiber, potassium, $\beta$-carotene equivalents, and vitamin $C$, had larger differences between the lowest and the highest score categories (Q1 and Q5) in the MOD2 scoring (Q5:Q1 ratios, 141-229\%). Saturated fatty acids showed similar trends across the three types of scorings; Q5:Q1 ratios in the ORG and MOD1 scores were 72\%, and that in the MOD2 score was 87\%.

Table 3. Median intake of food groups among quintile categories of the diet quality scores.

\begin{tabular}{|c|c|c|c|c|c|c|c|c|c|c|c|c|}
\hline & \multicolumn{4}{|c|}{ ORG Score } & \multicolumn{4}{|c|}{ MOD1 Score } & \multicolumn{4}{|c|}{ MOD2 Score } \\
\hline & Q1 & Q3 & Q5 & Q5:Q1 & Q1 & Q3 & Q5 & Q5:Q1 & Q1 & Q3 & Q5 & Q5:Q1 \\
\hline Grain, g/1000 kcal & 167 & 215 & 253 & $152 \%$ & 170 & 213 & 251 & $148 \%$ & 193 & 209 & 231 & $120 \%$ \\
\hline Vegetable, g/1000 kcal & 96 & 113 & 136 & $141 \%$ & 93 & 113 & 142 & $153 \%$ & 78 & 109 & 155 & $198 \%$ \\
\hline Fish and meat, g/1000 kcal & 125 & 107 & 104 & $83 \%$ & 125 & 107 & 104 & $84 \%$ & 102 & 107 & 125 & $122 \%$ \\
\hline Fish, g/1000 kcal & 31 & 26 & 24 & $80 \%$ & 30 & 26 & 25 & $83 \%$ & 23 & 27 & 30 & $132 \%$ \\
\hline Meat, g/1000 kcal & 39 & 33 & 31 & $80 \%$ & 39 & 33 & 31 & $80 \%$ & 36 & 32 & 36 & $101 \%$ \\
\hline Milk, g/1000 kcal & 132 & 92 & 82 & $62 \%$ & 133 & 91 & 82 & $62 \%$ & 44 & 102 & 124 & $282 \%$ \\
\hline Fruits, g/1000 kcal & 14 & 21 & 34 & $248 \%$ & 13 & 22 & 37 & $281 \%$ & 12 & 26 & 39 & $340 \%$ \\
\hline $\begin{array}{l}\text { Sugar and confectionaries, } \\
\mathrm{g} / 1000 \mathrm{kcal}\end{array}$ & 119 & 92 & 62 & $52 \%$ & 119 & 93 & 60 & $50 \%$ & 133 & 97 & 46 & $35 \%$ \\
\hline
\end{tabular}

ORG score, original score. MOD1 (first modified) score was calculated as scores with no upper limits for vegetable dishes and fruits. MOD2 (second modified) score was calculated as scores with no upper limits for all dishes except for sugar and confectionaries. Jonckheere-Terpstra tests were used to test the trend across the quintile categories of the scores, and all trends were significant $(p<0.001)$, except for the trend of meat intake across the MOD2 score $(p=0.874)$.

The scores of the three scorings were significantly associated with low glucose levels (all $p<0.001$; Table 5). The Akaike's information criterion was the lowest in the model with the MOD2 score as a dependent variable. Only the MOD2 scoring was significantly associated with low SBP $(-0.81 \mathrm{mmHg}$ per 10 points of the score; $p=0.001)$. The MS score was also significantly associated with the MOD2 scoring $(p=0.003)$; an increase in the score by 10 points was related to $0.89\left(\mathrm{e}^{-0.12}\right)$ times in the number of risks. 
Table 4. Median intake of nutrients among quintile categories of the diet quality scores.

\begin{tabular}{|c|c|c|c|c|c|c|c|c|c|c|c|c|}
\hline & \multicolumn{4}{|c|}{ ORG Score } & \multicolumn{4}{|c|}{ MOD1 Score } & \multicolumn{4}{|c|}{ MOD2 Score } \\
\hline & Q1 & Q3 & Q5 & Q5:Q1 & Q1 & Q3 & Q5 & Q5:Q1 & Q1 & Q3 & Q5 & Q5:Q1 \\
\hline Protein, $\%$ of total energy intake & 14.8 & 13.8 & 13.5 & $91 \%$ & 14.7 & 13.8 & 13.6 & $92 \%$ & 13.0 & 14.3 & 15.3 & $118 \%$ \\
\hline Fat, $\%$ of total energy intake & 34.0 & 30.2 & 27.1 & $80 \%$ & 34.2 & 30.1 & 27.1 & $79 \%$ & 32.0 & 29.6 & 28.6 & $89 \%$ \\
\hline SFA, $\%$ of total energy intake & 11.1 & 9.5 & 8.1 & $72 \%$ & 11.2 & 9.4 & 8.1 & $72 \%$ & 10.0 & 9.3 & 8.7 & $87 \%$ \\
\hline Carbohydrate, $\%$ of total energy intake & 49.2 & 54.1 & 57.8 & $117 \%$ & 49.1 & 54.2 & 57.9 & $118 \%$ & 53.4 & 54.6 & 54.6 & $102 \%$ \\
\hline Dietary fiber, g/1000 kcal & 4.7 & 5.3 & 5.9 & $125 \%$ & 4.6 & 5.2 & 6.1 & $131 \%$ & 4.4 & 5.8 & 6.3 & $142 \%$ \\
\hline Sodium, mg/1000 kcal & 1918 & 1807 & 1755 & $92 \%$ & 1902 & 1799 & 1774 & $93 \%$ & 1761 & 1843 & 1919 & $109 \%$ \\
\hline Potassium, mg/1000 kcal & 1172 & 1161 & 1203 & $103 \%$ & 1150 & 1163 & 1231 & $107 \%$ & 985 & 1255 & 1386 & $141 \%$ \\
\hline $\mathrm{Na} / \mathrm{K}$ ratio & 2.8 & 2.7 & 2.5 & $92 \%$ & 2.8 & 2.7 & 2.5 & $89 \%$ & 3.1 & 2.5 & 2.4 & $78 \%$ \\
\hline Calcium, mg/1000 kcal & 380 & 326 & 292 & $77 \%$ & 375 & 325 & 295 & $79 \%$ & 260 & 345 & 374 & $144 \%$ \\
\hline Magnesium, mg/1000 kcal & 119 & 117 & 123 & $104 \%$ & 117 & 117 & 124 & $106 \%$ & 105 & 127 & 137 & $131 \%$ \\
\hline Iron, $\mathrm{mg} / 1000 \mathrm{kcal}$ & 3.8 & 3.7 & 3.8 & $101 \%$ & 3.7 & 3.6 & 3.9 & $103 \%$ & 3.4 & 3.9 & 4.2 & $121 \%$ \\
\hline$\beta$ carotene equivalents, $\mu \mathrm{g} / 1000 \mathrm{kcal}$ & 1067 & 1291 & 1578 & $148 \%$ & 1025 & 1275 & 1687 & $165 \%$ & 829 & 1528 & 1896 & $229 \%$ \\
\hline Vitamin C, mg/1000 kcal & 44 & 51 & 61 & $140 \%$ & 42 & 51 & 64 & $152 \%$ & 38 & 59 & 68 & $176 \%$ \\
\hline Energy, kcal & 2044 & 2130 & 2184 & $107 \%$ & 2057 & 2127 & 2171 & $106 \%$ & 2062 & 2149 & 2016 & $98 \%$ \\
\hline
\end{tabular}

ORG score, original score. MOD1 (first modified) score was calculated as scores with no upper limits for vegetable dishes and fruits. MOD2 (second modified) score was calculated as scores with no upper limits for all dishes except for sugar and confectionaries. Jonckheere-Terpstra tests were used to test the trend across the quintile categories of the scores, and all trends were significant $(p<0.001)$, except for the trend of iron intake across the ORG score $(p=0.413)$ and energy intake across the MOD2 score $(p=0.130)$.

Table 5. Regression analysis of the effect of 10-point scores on cardiometabolic risks.

\begin{tabular}{ccccccccccccc}
\hline & \multicolumn{4}{c}{ ORG Score } & \multicolumn{4}{c}{ MOD1 Score } & \multicolumn{4}{c}{ MOD2 Score } \\
& $\boldsymbol{\beta}$ & SE & $\boldsymbol{p}$ & AIC & $\boldsymbol{\beta}$ & SE & $\boldsymbol{p}$ & AIC & $\boldsymbol{\beta}$ & SE & $\boldsymbol{p}$ & AIC \\
\hline zBMI $^{1}$ & -0.03 & 0.02 & 0.141 & 8456.6 & -0.03 & 0.02 & 0.133 & 8457 & -0.01 & 0.02 & 0.641 & 8459 \\
$\ln (\mathrm{TG}, \mathrm{mg} / \mathrm{dL})^{1}$ & 0.01 & 0.01 & 0.207 & 3601 & 0.01 & 0.01 & 0.249 & 3601 & -0.01 & 0.01 & 0.285 & 3601 \\
LDL-C, mg/DL $^{1}$ & -0.59 & 0.44 & 0.172 & 27,559 & -0.58 & 0.43 & 0.173 & 27,559 & -0.45 & 0.44 & 0.305 & 27,559 \\
HDL-C, mg/dL $^{1}$ & -0.49 & 0.30 & 0.106 & 25,285 & -0.57 & 0.30 & 0.057 & 25,284 & -0.16 & 0.31 & 0.603 & 25,287 \\
Glucose, mg/dL $^{1}$ & -0.46 & 0.13 & $<0.001$ & 19,949 & -0.45 & 0.13 & $<0.001$ & 19,949 & -0.50 & 0.13 & $<0.001$ & 19,947 \\
SBP, mmHg $^{1}$ & -0.09 & 0.25 & 0.710 & 24,120 & -0.12 & 0.25 & 0.633 & 24,120 & -0.81 & 0.25 & 0.001 & 24,110 \\
DBP, mmHg $^{1}$ & -0.02 & 0.20 & 0.936 & 22,542 & -0.01 & 0.19 & 0.963 & 22,542 & -0.24 & 0.20 & 0.235 & 22,541 \\
$\ln (\mathrm{MS} \mathrm{score})^{2}$ & -0.06 & 0.04 & 0.110 & 4264 & -0.07 & 0.04 & 0.085 & 4264 & -0.12 & 0.04 & 0.003 & 4258 \\
\hline
\end{tabular}

${ }^{1}$ Analyzed using the linear model. ${ }^{2}$ Analyzed using the Poisson model, with the number of cardiometabolic risks as a dependent variable. Both regression models were adjusted for age, sex, zBMI, sports activity, TV watching, sleep duration, number of siblings, and single parent. Models for zBMI as a dependent variable were adjusted for age, sex, sports activity, TV watching, sleep duration, number of siblings, and single parent. SE, standard error of a coefficient estimate ( $\beta$ ); AIC, Akaike's information criteria; zBMI, z-score of body mass index; TG, triglyceride; LDL-C and HDL-C, low- and high-density lipoprotein cholesterol, respectively; SBP and DBP; systolic and diastolic blood pressure, respectively. The MS score is the clustering of cardiometabolic risks.

\section{Discussion}

We examined three scoring types of dietary quality based on the JFGST. The contribution of each food group to the total score and the trends of nutrients and foods across the quintile categories were similar between the original and the first modified (ORG, and MOD1) scoring with no upper limits for vegetable dishes and fruits. In contrast, the contribution of fish and meat dishes and milk to the score was high in the MOD2 scoring with no upper limits for all dishes except for sugar and confectionaries. In addition, the MOD2 scoring and other scorings showed opposite trends for protein, sodium, and calcium intake, and the MOD2 scoring could differentiate the variance in dietary fiber, potassium, and vitamins compared with other scorings. All the three scoring types were significantly associated with the fasting glucose level. Notably, the MOD2 score was significantly negatively associated with SBP and the MS score.

Based on the data from the National Health and Nutrition Survey Japan, the modified score without upper limits for five dishes was negatively associated with SBP in adults $\geq 20$ years old of both sexes and with waist circumference in women [17]. In this study, only the modified score without upper limits for five dishes (MOD2 score) was associated with SBP and metabolic syndrome. However, in a study of female dietetic students aged 
18-22 years, the score was negatively associated with waist circumference and low-density lipoprotein cholesterol but was not associated with SBP [15]. Thus, the associations noted in female dietetic students were different from those in adolescents. A systematic review of diet quality indices developed for other countries showed associations with a reduced risk of cardiovascular disease and its risk factors [2]. Diet quality indices based on the JFGST were related to low all-cause and cerebrovascular disease mortality $[10,11]$. In adolescents, diet quality adherence to the JFGST may imply an association with low cardiometabolic risks. In particular, the MOD2 scoring without the upper limits for five dishes is a suitable index for high intake of dietary fiber, minerals, vitamins, fish, vegetables, fruits, and milk.

The higher the participants' MOD2 score points without upper limits for the five dishes, the higher their sodium intake, whereas using other scoring methods, the lower the sodium intake. Similar to other food frequency questionnaires, the BDHQ is vulnerable to measurement errors and is weak in estimating nutrients from foods that have not been investigated. Although sodium intake estimated from the BDHQ was barely associated with urinary sodium excretion as an intake biomarker, the sodium-to-potassium ratio from the BDHQ was significantly associated with the urinary ratio [30]. Furthermore, the sodiumto-potassium ratio was more closely associated with blood pressure than sodium intake in previous studies in youths $[37,38]$. In this study, the range of the sodium-to-potassium ratio across quintile categories was the widest in the scoring without upper limits for the five dishes. Thus, the association between the scoring without upper limits for five dishes and SBP, as well as the MS score, may be attributed to the sodium-to-potassium ratio.

Among the tentative dietary goals of preventing life-style related diseases, tentative dietary goals for sodium, saturated fatty acids, and a balance of energy-providing nutrients have upper limits of intake. Trends of saturated fatty acids across the quintile categories were similar among the three types of scorings, but the ORG and MOD1 score showed higher saturated fatty acids in the lowest categories than the MOD2 score did; this may reflect that the participants with intake above the optimum had low score points due to upper limits. However, the ORG and MOD1 scores did not show significant associations with cardiometabolic risk factors except for plasma glucose. The intake of two energy-providing nutrients (carbohydrates and protein) corresponds to servings of grain and fish and meat dishes in the JFGST. Two of the three energy-providing nutrients (fat being the third) can determine the nutritional balance. The tentative dietary goals for the energy-providing nutrients for the prevention of lifestyle-related diseases are 13-20\% for protein, 20-30\% for fat, and 50-65\% for carbohydrate according to the Dietary Reference Intakes for Japanese, 2020 [22]. Fat intake in the lowest categories of the three scores was above the tentative goals, but fat intake across the quintile categories was similar among the three scorings. The apparent difference in the trends across the quintile categories among the three scores was protein, although total protein is not considered a pivotal determinant of the nutritional balance for the tentative dietary goals to prevent lifestyle-related diseases [22]. Thus, nutrients other than those providing energy may determine the association between the score and cardiometabolic risks. A rice grain dish is the main staple of the Japanese diet $[8,39]$, but this may not be a main source of health benefits.

The ORG scores with upper limits for vegetable dishes and fruits, and the MOD1 scores without upper limits had similar points for these food groups and dietary nutrient patterns. This means that the proportion of participants with vegetable and fruit intake above the upper limits was low; thus, this population could not ascertain the beneficial effects of vegetable and fruit intake in the JFGST scoring. The Health Japan 21 (the second term) program recommends a mean daily intake of vegetables $\geq 350 \mathrm{~g}$, which represents a notable increase from $282 \mathrm{~g}$ in 2010. In addition, it aims to reduce the proportion of individuals who consume fruits $<100 \mathrm{~g}$ from $61.4 \%$ in 2010 to 30\% [22]. The MOD2 scoring, which showed large differences of vegetables and fruits between the lowest and highest scores, was negatively associated with metabolic risks. The MOD2 scoring results suggest limited evidence to support an intervention to increase adolescents' consumption of vegetables and fruits from their current intake. However, rather than focusing on a single food group, 
such as vegetables and fruits, consuming a healthy balance of food should be considered for Japanese adolescents.

Similar to previous dietary indices associated with cardiovascular diseases rather than cancers [1,2], the JFGST was associated with cardiometabolic risk factors. The JFGST was developed in consideration of the concept of Japanese culture. Japanese terms for dishes are derived from ichiju-sansai, a common formula of Japanese cuisine, Washoku. Grain dishes, shushoku, are the main staples; fish and meat dishes, shusai, are the main dishes; and vegetable dishes, fukusai, are the side dishes [40]. The concept of Japanese dishes is disseminated through homemaking classes and nutrition education at school. The JFGST is a food-based index unlike most of other existing dietary indices [41]. The Healthy Eating Index, the Diet Quality Index, and the Mediterranean Diet Quality Score use foods and nutrients together as scoring items [3-5]. The Mediterranean Diet Quality Index for children and adolescents uses food intake and dietary habits, but this scoring may be influenced by culture [6]. When using the JFGST as a health promotion and education tool, adolescents can easily understand their suitable dish servings. For effective nutrition education, the dietary index should be suited to the background of the target population.

In contrast to the scoring items, the JFGST scoring uses a combination of nutrient and food intake; the servings of grain dishes, fish and meat dishes, and milk were calculated from nutrient amount, but the servings of vegetable dishes and fruits were from the food amount. It is unknown whether servings that adolescents have in mind coincide with those of the JFGST scoring. Dietary indices have three objectives: to measure absolute diet quality, to evaluate adherence to dietary guidelines, and to guide health promotion [41]. According to the third objective, Japanese youths should be educated on favorable dietary habits to ensure longevity and low mortality. Practical use of existing dietary indices for health promotion should be addressed [1,2], and the JFGST should be adopted for nutrition education interventions.

This study had some limitations. The cross-sectional design of this study could not explain temporal causality between the scores and cardiometabolic risk factors, but the participants might not have known their own cardiovascular parameter levels before answering the BDHQ15y. The design could also not explore the effect on risks appearing in later life, such as cardiovascular mortality, cancer incidence, frailty, and dementia related to healthy life expectancy. Social norms regarding a healthy diet may contribute to a reporting bias, but this may attenuate observable associations. The BDHQ15y has weaknesses, similar to other frequent food questionnaires. Intrinsic measurement errors and biases of the BDHQ15y may attenuate or mask the true associations between the score and cardiovascular risk. Nevertheless, the associations found in this study could help adolescents whose diets are assessed using the BDHQ15y to review and modify their own dietary habits with the JFGST score. Another limitation is that the study location was limited to a small part of Japan, meaning that the data collection could not capture all Japanese dietary patterns.

\section{Conclusions}

We showed for the first time that Japanese adolescents adherent to the JFGST had low cardiovascular risk. However, only the modified JFGST scoring system with no upper limits for five food groups (grain dishes, fish and meat dishes, vegetable dishes, milk, and fruits) was beneficial in alleviating cardiovascular risks; this indicates that adolescents should not fall below the lower limits for intake of the abovementioned five food groups. The JFGST can be used for adolescent health education.

Supplementary Materials: The following are available online at https://www.mdpi.com/article/10 .3390/nu14010043/s1, Table S1: Food groups in the Japanese Food Guide Spinning Top and their optimal ranges, Figure S1: Histogram and scores for grain and vegetable dishes, Figure S2: Histogram and scores for fish and meat dishes and milk, Figure S3: Histogram and scores for fruits, and sugar and confectionaries. 


\begin{abstract}
Author Contributions: M.O., A.F. and S.S. contributed to the conception of the study. M.O. contributed to the study design. M.O. organized the database. S.S. calculated the food and nutrient intake from the BDHQ. M.O. performed statistical analyses. M.O. wrote the first draft of this manuscript. All authors contributed to manuscript revision. All authors have read and agreed to the published version of the manuscript.
\end{abstract}

Funding: This study was funded by the Japan Society for the Promotion of Science (grant numbers JP18K10085 and JP21K10497).

Institutional Review Board Statement: The study was conducted according to the guidelines of the Declaration of Helsinki, and all procedures involving human subjects were approved by the Institutional Review Board of Yamaguchi University Hospital (H17-14-2).

Informed Consent Statement: Complying with the Japanese ethical guideline, informed assent was obtained from all participants 13 and 14 years old, and written informed consent was obtained from the guardians of all participants.

Data Availability Statement: The raw data supporting the conclusions of this article will be made available by the corresponding author without undue reservation.

Acknowledgments: We appreciate the collaboration of the health center, the education board, and schools of Shunan City. We thank the study participants and their guardians. We would also like to thank Ichiro Kunitsugu, Shin-ichi Sugiyama, and Norikazu Yoshitake for their help with the survey.

Conflicts of Interest: The authors declare that the research was conducted in the absence of any commercial or financial relationships that could be construed as a potential conflict of interest.

\title{
References
}

1. Kant, A.K. Dietary patterns and health outcomes. J. Am. Diet. Assoc. 2004, 104, 615-635. [CrossRef]

2. Wirt, A.; Collins, C.E. Diet quality-What is it and does it matter? Public Health Nutr. 2009, 12, 2473-2492. [CrossRef] [PubMed]

3. Patterson, R.E.; Haines, P.S.; Popkin, B.M. Diet quality index: Capturing a multidimensional behavior. J. Am. Diet Assoc. 1994, 94, 57-64. [CrossRef]

4. Krebs-Smith, S.M.; Pannucci, T.E.; Subar, A.F.; Kirkpatrick, S.I.; Lerman, J.L.; Tooze, J.A.; Wilson, M.M.; Reedy, J. Update of the Healthy Eating Index: HEI-2015. J. Acad. Nutr. Diet. 2018, 118, 1591-1602. [CrossRef] [PubMed]

5. Trichopoulou, A.; Kouris-Blazos, A.; Wahlqvist, M.L.; Gnardellis, C.; Lagiou, P.; Polychronopoulos, E.; Vassilakou, T.; Lipworth, L.; Trichopoulos, D. Diet and overall survival in elderly people. BMJ 1995, 311, 1457-1460. [CrossRef]

6. Serra-Majem, L.; Ribas, L.; Garcia, A.; Perez-Rodrigo, C.; Aranceta, J. Nutrient adequacy and Mediterranean Diet in Spanish school children and adolescents. Eur. J. Clin. Nutr. 2003, 57 (Suppl. S1), S35-S39. [CrossRef] [PubMed]

7. Ministry of Agriculture Forestry and Fisheries. Food Supply and Demand Table. 2019. Available online: https://www.maff.go. $\mathrm{jp} / \mathrm{j} /$ zyukyu/fbs/ (accessed on 15 October 2021).

8. Suzuki, N.; Goto, Y.; Ota, H.; Kito, K.; Mano, F.; Joo, E.; Ikeda, K.; Inagaki, N.; Nakayama, T. Characteristics of the Japanese diet described in epidemiologic publications: A qualitative systematic review. J. Nutr. Sci. Vitaminol. 2018, 64, 129-137. [CrossRef]

9. Yoshiike, N.; Hayashi, F.; Takemi, Y.; Mizoguchi, K.; Seino, F. A new food guide in Japan: The Japanese food guide Spinning Top. Nutr. Rev. 2007, 65, 149-154. [CrossRef]

10. Oba, S.; Nagata, C.; Nakamura, K.; Fujii, K.; Kawachi, T.; Takatsuka, N.; Shimizu, H. Diet based on the Japanese Food Guide Spinning Top and subsequent mortality among men and women in a general Japanese population. J. Am. Diet. Assoc. 2009, 109, 1540-1547. [CrossRef]

11. Kurotani, K.; Akter, S.; Kashino, I.; Goto, A.; Mizoue, T.; Noda, M.; Sasazuki, S.; Sawada, N.; Tsugane, S.; Japan Public Health Center Based Prospective Study Group. Quality of diet and mortality among Japanese men and women: Japan Public Health Center based prospective study. BMJ 2016, 352, i1209. [CrossRef]

12. Kuriyama, N.; Murakami, K.; Livingstone, M.B.E.; Okubo, H.; Kobayashi, S.; Suga, H.; Sasaki, S. Development of a food-based diet quality score for Japanese: Associations of the score with nutrient intakes in young, middle-aged and older Japanese women. J. Nutr. Sci. 2016, 5, e41. [CrossRef] [PubMed]

13. Sakai, H.; Murakami, K.; Kobayashi, S.; Suga, H.; Sasaki, S. Food-based diet quality score in relation to depressive symptoms in young and middle-aged Japanese women. Br. J. Nutr. 2017, 117, 1674-1681. [CrossRef] [PubMed]

14. Okubo, R.; Matsuoka, Y.J.; Sawada, N.; Mimura, M.; Kurotani, K.; Nozaki, S.; Shikimoto, R.; Tsugane, S. Diet quality and depression risk in a Japanese population: The Japan Public Health Center (JPHC)-based Prospective Study. Sci. Rep. 2019, 9, 7150. [CrossRef] [PubMed]

15. Nishimura, T.; Murakami, K.; Livingstone, M.B.; Sasaki, S.; Uenishi, K. Adherence to the food-based Japanese dietary guidelines in relation to metabolic risk factors in young Japanese women. Br. J. Nutr. 2015, 114, 645-653. [CrossRef] 
16. Nanri, A.; Nagai, C.; Kochi, T.; Eguchi, M.; Kabe, I.; Mizoue, T. Diet quality and depressive symptoms among workers. Clin. Nutr. 2020, 39, 1951-1957. [CrossRef]

17. Murakami, K.; Livingstone, M.B.E.; Sasaki, S. Diet quality scores in relation to metabolic risk factors in Japanese adults: A cross-sectional analysis from the 2012 National Health and Nutrition Survey, Japan. Eur. J. Nutr. 2019, 58, 2037-2050. [CrossRef]

18. Yamamoto, K.; Ota, M.; Minematsu, A.; Motokawa, K.; Yokoyama, Y.; Yano, T.; Watanabe, Y.; Yoshizaki, T. Association between adherence to the Japanese Food Guide Spinning Top and sleep quality in college students. Nutrients 2018, 10, 1996. [CrossRef]

19. Shinsugi, C.; Tani, Y.; Kurotani, K.; Takimoto, H.; Ochi, M.; Fujiwara, T. Change in growth and diet quality among preschool children in Tokyo, Japan. Nutrients 2020, 12, 1290. [CrossRef]

20. Hashimoto, A.; Murakami, K.; Kobayashi, S.; Suga, H.; Sasaki, S. Associations of education with overall diet quality are explained by different food groups in middle-aged and old Japanese women. J. Epidemiol. 2021, 31, 280-286. [CrossRef]

21. Osaki, Y.; Minowa, M.; Suzuki, K.; Wada, K. Nationwide survey on alcohol use among junior and senior high school students in Japan. Jpn. J. Public Health 1999, 46, 883-893.

22. Minister of Health Labour and Welfare. Health Japan 21 (The Second Term). Available online: https://www.mhlw.go.jp/stf/ seisakunitsuite/bunya/kenkou_iryou/kenkou/kenkounippon21.html (accessed on 25 October 2021).

23. Shinozaki, K.; Okuda, M.; Sasaki, S.; Kunitsugu, I.; Shigeta, M. Dietary fiber consumption decreases the risks of overweight and hypercholesterolemia in Japanese children. Ann. Nutr. Metab. 2015, 67, 58-64. [CrossRef]

24. Okuda, M.; Fujiwara, A.; Sasaki, S. Added and free sugars intake and metabolic biomarkers in Japanese adolescents. Nutrients 2020, 12, 2046. [CrossRef] [PubMed]

25. Okuda, M.; Fujiwara, A.; Sasaki, S. Composition of dietary fatty acids and health risks in Japanese youths. Nutrients 2021, 13, 426 [CrossRef]

26. Kobayashi, S.; Murakami, K.; Sasaki, S.; Okubo, H.; Hirota, N.; Notsu, A.; Fukui, M.; Date, C. Comparison of relative validity for food group intake estimated by comprehensive and brief-type self-administered diet history questionnaires against 16-day dietary records in Japanese adults. Public Health Nutr. 2011, 14, 1200-1211. [CrossRef] [PubMed]

27. Kobayashi, S.; Honda, S.; Murakami, K.; Sasaki, S.; Okubo, H.; Hirota, N.; Notsu, A.; Fukui, M.; Date, C. Both comprehensive and brief self-administered diet history questionnaires satisfactorily rank nutrient intakes in Japanese adults. J. Epidemiol. 2012, 22, 151-159. [CrossRef] [PubMed]

28. Okuda, M.; Sasaki, S.; Bando, N.; Hashimoto, M.; Kunitsugu, I.; Sugiyama, S.; Terao, J.; Hobara, T. Carotenoid, tocopherol, and fatty acid biomarkers and dietary intake estimated by using a brief self-administered diet history questionnaire for older Japanese children and adolescents. J. Nutr. Sci. Vitaminol. 2009, 55, 231-241. [CrossRef] [PubMed]

29. Okuda, M.; Asakura, K.; Sasaki, S. Protein intake estimated from brief-type self-administered diet history questionnaire and urinary urea nitrogen level in adolescents. Nutrients 2019, 11, 319. [CrossRef]

30. Okuda, M.; Sasaki, S. Assessment of foods associated with sodium and potassium intake in Japanese youths using the brief-type self-administered diet history questionnaire. Nutrients 2021, 13, 2345. [CrossRef] [PubMed]

31. Sasaki, S.; Katagiri, A.; Tsuji, T.; Shimoda, T.; Amano, K. Self-reported rate of eating correlates with body mass index in 18-y-old Japanese women. Int. J. Obes. Relat. Metab. Disord. 2003, 27, 1405-1410. [CrossRef] [PubMed]

32. Willet, W. Nutritional Epidemiology, 3rd ed.; Oxford University Press: New York, NY, USA, 2013; ISBN 978-0-19-975403-8.

33. Ministry of Health Laboror and Wealfare. Report on Utilization of Dietary Reference Intakes for Japanese. Available online: https:/ / www.mhlw.go.jp/shingi/2010/03/s0331-9.html (accessed on 25 October 2021).

34. Kato, N.; Takimoto, H.; Sudo, N. The cubic functions for spline smoothed L, S and M values for BMI reference data of Japanese children. Clin. Pediatr. Endocrinol. 2011, 20, 47-49. [CrossRef]

35. Cole, T.J.; Green, P.J. Smoothing reference centile curves: The LMS method and penalized likelihood. Stat. Med. 1992, 11, 1305-1319. [CrossRef] [PubMed]

36. R Core Team. R: A Language and Environment for Statistical Computing. Available online: https://www.R-project.org/ (accessed on 1 December 2020).

37. Geleijnse, J.M.; Grobbee, D.E.; Hofman, A. Sodium and potassium intake and blood pressure change in childhood. BMJ 1990, 300, 899-902. [CrossRef] [PubMed]

38. Buendia, J.R.; Bradlee, M.L.; Daniels, S.R.; Singer, M.R.; Moore, L.L. Longitudinal effects of dietary sodium and potassium on blood pressure in adolescent girls. JAMA Pediatr. 2015, 169, 560-568. [CrossRef] [PubMed]

39. Ministry of Agriculture Forestry and Fisheries. Washoku: Traditional Dietary Cultures of the Japanese. Available online: https://www.maff.go.jp/e/data/publish/attach/pdf/index-20.pdf (accessed on 15 October 2021).

40. Ministry of Agriculture Forestry and Fisheries. The Washoku Way Japan's Nuanced Approach to Food. Available online: https://www.maff.go.jp/j/shokusan/gaisyoku/pamphlet/ (accessed on 15 December 2021).

41. Waijers, P.M.; Feskens, E.J.; Ocke, M.C. A critical review of predefined diet quality scores. Br. J. Nutr. 2007, 97, 219-231. [CrossRef] [PubMed] 